\title{
Nonlinear stochastic analysis for a stochastic SIS epidemic model
}

\author{
Fei Li ${ }^{\mathrm{a}}$, Xinzhu Menga, ${ }^{\mathrm{a}, \mathrm{b}, *}$, Yujun Cui ${ }^{\mathrm{a}}$ \\ ${ }^{a}$ College of Mathematics and Systems Science, Shandong University of Science and Technology, Qingdao 266590, P. R. China. \\ ${ }^{b}$ State Key Laboratory of Mining Disaster Prevention and Control Co-founded by Shandong Province and the Ministry of Science and \\ Technology, Shandong University of Science and Technology, Qingdao 266590, P. R. China.
}

Communicated by X. Z. Liu

\begin{abstract}
This paper considers a stochastic Susceptible-Infected-Susceptible (SIS) epidemic model with nonlinear saturated incidence. The threshold conditions for disease extinction and stochastic permanence are obtained by using nonlinear stochastic analysis for Feller's test and the canonical probability method. Consequently, this improves and extends some previous results obtained by using Lyapunov method. A series of numerical simulations are carried out to illustrate our theoretical findings. (C)2017 All rights reserved.
\end{abstract}

Keywords: Stochastic SIS epidemic model, Feller's test, stochastic permanence, nonlinear saturated incidence. 2010 MSC: 60H10, 65C30, 91B70.

\section{Introduction}

Mathematical models for the dynamics of infectious diseases have a realistic significance in the field of epidemiology, and some novel results are obtained [1-3, 5, 7, 13, 17, 20-22, 29-31]. Recently, nonlinear analysis has been widely applied in mathematical modelling in biology [2, 3, 5, 13, 21, 22, 31]. Inspired by previous work for stochastic dynamics in $[9,10,12,14,16,19,23,24,26-28,32]$, this paper discusses a nonlinear SIS epidemic model with stochastic perturbation, which is described by the following stochastic system

$$
\left\{\begin{array}{l}
\mathrm{d} S(\mathrm{t})=\left(\Lambda-\mu S(t)-\frac{\beta S(t) I(t)}{1+\alpha I(t)}+\lambda I(t)\right) d t-\frac{\sigma S(t) I(t)}{1+\alpha I(t)} d B(t), \\
d I(t)=\left[\frac{\beta S(t) I(t)}{1+\alpha I(t)}-(\mu+\lambda) I(t)\right] d t+\frac{\sigma S(t) I(t)}{1+\alpha I(t)} d B(t),
\end{array}\right.
$$

where $S(t)$ and $I(t)$, respectively, represent the number of susceptible and infectious individuals at time $t, B(t)(B(0)=0)$ is the standard Wiener process with intensity $\sigma^{2}>0, \wedge$ stands for the birth rate of susceptible individuals, $\mu$ denotes the natural mortality, $\lambda$ is the recovery rate of disease. The function $\frac{\beta S(t) I(t)}{1+\alpha I(t)}$ stands for the saturated incidence rate, here $\beta$ represents the transmission rate of disease and $\alpha$

\footnotetext{
*Corresponding author

Email addresses: lifei930512@163.com (Fei Li), mxz721106@sdust.edu.cn (Xinzhu Meng), cyj720201@163.com (Yujun Cui)
} doi:10.22436/jnsa.010.09.47 
is the half-saturation constant. The parameters $\beta, \alpha, \lambda$ are all nonnegative constants and $\Lambda, \mu$ are positive constants. Recently, authors of $[4,6,21]$ have done much research and have obtained plenty of significant conclusions for system (1.1). They set up the threshold condition $\mathrm{R}^{*}$, which determines disease extinction or permanence under stochastic perturbation, where

$$
R^{*}=R-\frac{\sigma^{2} \Lambda^{2}}{2 \mu^{2}(\mu+\lambda)}
$$

and $R=\frac{\beta \Lambda}{\mu(\mu+\lambda)}$ stands for the basic reproduction of its deterministic system. Meanwhile, they provided the results as follows:

(A1) For any given initial value $(S(0), I(0)) \in \mathbb{R}_{+}^{2}$, the system (1.1) has a unique positive solution $(\mathrm{S}(\mathrm{t}), \mathrm{I}(\mathrm{t})) \in \mathbb{R}_{+}^{2}$ on $\mathrm{t} \geqslant 0$ a.s.

(A2) When $R^{*}<1$ and $\sigma^{2} \leqslant \frac{\beta \mu}{\Lambda}$, it shows that the disease dies out with probability one.

(A3) When $R^{*}>1$, it shows that the disease is permanent in mean with probability one.

From system (1.1), we have

$$
\frac{d(S(t)+I(t))}{d t}=\Lambda-\mu(S(t)+I(t))
$$

This implies that

$$
\lim _{t \rightarrow+\infty}(S(t)+I(t))=\frac{\Lambda}{\mu} .
$$

Thus the one-dimensional limiting system of system (1.1) is given by

$$
\mathrm{dI}(\mathrm{t})=\left[\frac{\beta(\Lambda-\mu \mathrm{I}(\mathrm{t})) \mathrm{I}(\mathrm{t})}{\mu(1+\alpha \mathrm{I}(\mathrm{t}))}-(\mu+\lambda) \mathrm{I}(\mathrm{t})\right] \mathrm{dt}+\frac{\sigma(\Lambda-\mu \mathrm{I}(\mathrm{t})) \mathrm{I}(\mathrm{t})}{\mu(1+\alpha \mathrm{I}(\mathrm{t}))} \mathrm{dB}(\mathrm{t}),
$$

with initial value $I(0) \in\left(0, \frac{\Lambda}{\mu}\right)$ and then we concentrate on exploring the dynamics of system (1.2).

For another, a large number of literatures have used the Lyapunov functions to study the dynamics of infectious diseases and have given many good results $[4,6,11,21]$. However, this paper applies the Feller's test and canonical probability methods to study the dynamics of system (1.2) and gives some new results. In this paper, we are mainly concerned with the following three questions:

(Q1) Removing the condition of $\sigma^{2} \leqslant \frac{\beta \mu}{\Lambda}$, if $R^{*}<1$ holds, will still the disease go extinct?

(Q2) If $\mathrm{R}^{*}=1$ holds, will the disease die out or persist?

(Q3) If $R^{*}>1$ holds, is system (1.2) stochastic permanence in addition to permanence in mean?

The remainder of this paper is organized as follows. In Section 2, we obtain the conditions of disease extinction by Feller's test: $R^{*}<1$ or $R^{*}=1$ is the sufficient condition of disease extinction with probability one. In Section 3, we study the stochastic permanence of the disease via Chebyshev inequality which is different from the previous results about permanence in mean. Finally, we show the main results and then present some numerical simulations to illustrate the main results.

Throughout this paper, unless otherwise specified, let $\left(\Omega, \mathscr{F},\{\mathscr{F}\}_{\mathrm{t} \geqslant 0}, \mathbb{P}\right)$ be a complete probability space with a filtration $\left\{\mathscr{F}_{t}\right\}_{t} \geqslant 0$ satisfying the usual conditions (i.e., it is increasing and right continuous while $\mathscr{F}_{0}$ contains all $\mathbb{P}$-null sets). Further suppose $\mathrm{B}(\mathrm{t})$ is defined on the complete probability space $\Omega$.

\section{Extinction}

In this section, we mainly investigate the dynamics of system (1.2) for $R^{*}<1$ and $R^{*}=1$, respectively, and give some new results. Now let us state an assumption and some lemmas of a result on the Feller's test $[8,25]$ which will be useful in proving the main results. 
Assumption 2.1. Assume that the following one-dimensional time-homogeneous stochastic differential equation (SDE) [18]:

$$
d X(t)=f(X(t)) d t+g(X(t)) d B(t), X(0) \in \mathbb{R}_{+},
$$

satisfying the following properties:

(i) $\forall X \in Y, \quad g^{2}(X)>0$;

(ii) $\forall \mathrm{X} \in \mathrm{Y}, \quad \exists \epsilon>0, \quad \int_{X-\epsilon}^{X+\epsilon} \frac{1+|\mathrm{f}(\mathrm{r})|}{\mathrm{g}^{2}(\mathrm{r})} \mathrm{dr}<+\infty$,

here $Y=(\xi, \gamma)$ and $-\infty \leqslant \xi<\gamma \leqslant+\infty$.

Lemma 2.2. If Assumption 2.1 holds, then let $\mathrm{X}(\mathrm{t})$ be a non-explosive solution of the above SDE $(2.1)$ in $\mathrm{Y}=(\xi, \gamma)$. For any fixed constant $\mathrm{c} \in \mathrm{Y}$, the scale function is described by

$$
\zeta(x)=\int_{c}^{x} \exp \left\{-2 \int_{c}^{u} \frac{f(r)}{g^{2}(r)} d r\right\} d u .
$$

When $\zeta\left(\xi^{+}\right)>-\infty, \zeta\left(\gamma^{-}\right)=+\infty$, then

$$
\mathbb{P}\left\{\lim _{t \rightarrow+\infty} X(t)=\xi\right\}=\mathbb{P}\left\{\sup _{t \geqslant 0} X(t)<\gamma\right\}=1 .
$$

Lemma 2.3. Assume that the one-dimensional $\operatorname{SDE}\left(d \mathrm{X}(\mathrm{t})=\mathrm{f}(\mathrm{X}(\mathrm{t})) d \mathrm{t}+d \mathrm{~B}(\mathrm{t}), \mathrm{X}(0) \in \mathbb{R}_{+}\right)$exists a nonexplosive solution in finite time which is unique in the sense of probability law.

Define

$$
\alpha(x)=\int_{0}^{x} \exp \left\{2 \int_{0}^{u} f(r) d r\right\} d u, \quad \beta(x)=\int_{0}^{x} \exp \left\{-2 \int_{0}^{\mathfrak{u}} f(r) d r\right\} d u .
$$

When $\alpha(+\infty)<+\infty, \alpha(-\infty)=-\infty, \beta(+\infty)=+\infty, \beta(-\infty)=-\infty$, then

$$
\forall \Gamma \in \mathbb{R}, \quad \mathbb{P}\left\{\lim _{\mathrm{t} \rightarrow+\infty} X(\mathrm{t})<\Gamma\right\}=1,
$$

that is to say, $\mathrm{X}(\mathrm{t}) \rightarrow-\infty$ in probability meaning.

Proof. The proof of Lemma 2.2 and Lemma 2.3 are similar to $[8,25]$, hence we omit it here.

Theorem 2.4. Let $\mathrm{I}(\mathrm{t})$ be the solution of system (1.2) with initial value $\mathrm{I}(0) \in\left(0, \frac{\Lambda}{\mu}\right)$. If $\mathrm{R}^{*}<1$ holds, then $\mathbb{P}\left\{\lim _{t \rightarrow+\infty} I(t)=0\right\}=1$.

Proof. By virtue of Lemma 2.2 and system (1.2), let

$$
f(I)=\frac{\beta(\Lambda-\mu I) I}{\mu(1+\alpha I)}-(\mu+\lambda) I, \quad g(I)=\frac{\sigma(\Lambda-\mu I) I}{\mu(1+\alpha I)} .
$$

It is easy to get that

$$
\begin{aligned}
2 \int_{c}^{u} \frac{f(r)}{g^{2}(r)} d r= & \frac{2}{\sigma^{2}} \int_{c}^{u}\left[\frac{\Lambda \mu \beta+\Lambda^{2} \beta \alpha-\mu^{2}(\mu+\lambda)+\Lambda^{2}(\mu+\lambda) \alpha^{2}}{\Lambda^{2}\left(\frac{\Lambda}{\mu}-r\right)}+\frac{\Lambda \mu \beta-\mu^{2}(\mu+\lambda)}{\Lambda^{2} r}\right. \\
& \left.-\frac{\mu^{2}(\mu+\lambda)+2 \mu \Lambda(\mu+\lambda) \alpha+\Lambda^{2}(\mu+\lambda) \alpha^{2}}{\mu \Lambda\left(\frac{\Lambda}{\mu}-r\right)^{2}}\right] d r \\
= & -\frac{2}{\sigma^{2}}\left[\frac{\Lambda \mu \beta+\Lambda^{2} \beta \alpha-\mu^{2}(\mu+\lambda)+\Lambda^{2}(\mu+\lambda) \alpha^{2}}{\Lambda^{2}} \ln \left(\frac{\Lambda}{\mu}-u\right)-\ln u\right. \\
& \left.\times \frac{\Lambda \mu \beta-\mu^{2}(\mu+\lambda)}{\Lambda^{2}}+\frac{\mu^{2}(\mu+\lambda)+2 \mu \Lambda(\mu+\lambda) \alpha+\Lambda^{2}(\mu+\lambda) \alpha^{2}}{\Lambda(\Lambda-\mu u)}\right]+M_{c}
\end{aligned}
$$


here $M_{c}$ is a constant. Then by calculation, one can obtain

$$
\begin{aligned}
\zeta(I)= & \exp \left\{-M_{c}\right\} \int_{c}^{I} \exp \left\{\frac { 2 } { \sigma ^ { 2 } } \left[\frac{\Lambda \mu \beta+\Lambda^{2} \beta \alpha-\mu^{2}(\mu+\lambda)+\Lambda^{2}(\mu+\lambda) \alpha^{2}}{\Lambda^{2}} \ln \left(\frac{\Lambda}{\mu}-u\right)\right.\right. \\
& \left.\left.-\frac{\Lambda \mu \beta-\mu^{2}(\mu+\lambda)}{\Lambda^{2}} \ln u+\frac{\mu^{2}(\mu+\lambda)+2 \mu \Lambda(\mu+\lambda) \alpha+\Lambda^{2}(\mu+\lambda) \alpha^{2}}{\Lambda(\Lambda-\mu u)}\right]\right\} d u \\
= & \exp \left\{-M_{c}\right\} \int_{c}^{I} u u^{-\frac{2}{\sigma^{2}}\left[\frac{\Lambda \mu \beta-\mu^{2}(\mu+\lambda)}{\Lambda^{2}}\right]}\left(\frac{\Lambda}{\mu}-u\right)^{\frac{2}{\sigma^{2}}\left[\frac{\Lambda \mu \beta+\Lambda^{2} \beta \alpha-\mu^{2}(\mu+\lambda)+\Lambda^{2}(\mu+\lambda) \alpha^{2}}{\Lambda^{2}}\right]} \\
& \times \exp \left\{\frac{2\left[\mu^{2}(\mu+\lambda)+2 \mu \Lambda(\mu+\lambda) \alpha+\Lambda^{2}(\mu+\lambda) \alpha^{2}\right]}{\sigma^{2} \Lambda(\Lambda-\mu u)}\right\} d u .
\end{aligned}
$$

Now from (2.2), let $I \rightarrow\left(\frac{\Lambda}{\mu}\right)^{-}, \rho=\frac{\mu}{\Lambda-\mu u}$, we have

$$
\begin{aligned}
\zeta\left(\left(\frac{\Lambda}{\mu}\right)^{-}\right) \geqslant & \exp \left\{-M_{c}\right\} c^{\frac{2 \mu^{2}(\mu+\lambda)}{\sigma^{2} \Lambda^{2}}}\left(\frac{\Lambda}{\mu}\right)^{-\frac{2 \mu \beta}{\sigma^{2} \Lambda}} \int_{\frac{\mu}{\Lambda-\mu c}}^{+\infty} \rho^{-\frac{2}{\sigma^{2}}\left[\frac{\Lambda \mu \beta+\Lambda^{2} \beta \alpha-\mu^{2}(\mu+\lambda)+\Lambda^{2}(\mu+\lambda) \alpha^{2}}{\Lambda^{2}}\right]-2} \\
& \times \exp \left\{\frac{2\left[\mu^{2}(\mu+\lambda)+2 \mu \Lambda(\mu+\lambda) \alpha+\Lambda^{2}(\mu+\lambda) \alpha^{2}\right] \rho}{\sigma^{2} \Lambda \mu}\right\} d \rho \\
= & +\infty .
\end{aligned}
$$

Notice that $R^{*}<1$ such that $\frac{2}{\sigma^{2}}\left[\frac{\Lambda \mu \beta-\mu^{2}(\mu+\lambda)}{\Lambda^{2}}\right]<1$, then from (2.2) and let $\mathrm{I} \rightarrow 0^{+}$, one sees that

$$
\begin{aligned}
-\zeta\left(0^{+}\right) \leqslant & \exp \left\{-M_{c}\right\}\left(\frac{\Lambda}{\mu}\right)^{\frac{2\left[\Lambda \mu \beta+\Lambda^{2} \beta \alpha+\Lambda^{2}(\mu+\lambda) \alpha^{2}\right]}{\sigma^{2} \Lambda^{2}}}\left(\frac{\Lambda}{\mu}-c\right)^{-\frac{2 \mu^{2}(\mu+\lambda)}{\sigma^{2} \Lambda^{2}}} \\
& \times \exp \left\{\frac{2\left[\mu^{2}(\mu+\lambda)+2 \mu \Lambda(\mu+\lambda) \alpha+\Lambda^{2}(\mu+\lambda) \alpha^{2}\right]}{\sigma^{2} \Lambda(\Lambda-\mu c)}\right\} \int_{0}^{c} u^{-\frac{2}{\sigma^{2}}\left[\frac{\Lambda \mu \beta-\mu^{2}(\mu+\lambda)}{\Lambda^{2}}\right]} d u \\
= & \exp \left\{-M_{c}\right\}\left(\frac{\Lambda}{\mu}\right)^{\frac{2\left[\Lambda \mu \beta+\Lambda^{2} \beta \alpha+\Lambda^{2}(\mu+\lambda) \alpha^{2}\right]}{\sigma^{2} \Lambda^{2}}}\left(\frac{\Lambda}{\mu}-c\right)^{-\frac{2 \mu^{2}(\mu+\lambda)}{\sigma^{2} \Lambda^{2}}} \\
& \times \exp \left\{\frac{2\left[\mu^{2}(\mu+\lambda)+2 \mu \Lambda(\mu+\lambda) \alpha+\Lambda^{2}(\mu+\lambda) \alpha^{2}\right]}{\sigma^{2} \Lambda(\Lambda-\mu c)}\right\} \\
& \times \frac{1}{1-\frac{2}{\sigma^{2}}\left[\frac{\Lambda \mu \beta-\mu^{2}(\mu+\lambda)}{\Lambda^{2}}\right]} c^{1-\frac{2}{\sigma^{2}}\left[\frac{\Lambda \mu \beta-\mu^{2}(\mu+\lambda)}{\Lambda^{2}}\right]} \\
< & +\infty,
\end{aligned}
$$

which implies that $\zeta\left(0^{+}\right)>-\infty$. The proof of Theorem 2.4 is complete.

Theorem 2.5. Let $\mathrm{I}(\mathrm{t})$ be the solution of system (1.2) with initial value $\mathrm{I}(0) \in\left(0, \frac{\Lambda}{\mu}\right)$. If $\mathrm{R}^{*}=1$ holds, then $\mathrm{t} \rightarrow+\infty, \mathrm{I}(\mathrm{t}) \rightarrow 0$ in probability meaning.

Proof. Define

$$
\mathrm{V}=\eta(\mathrm{I})=\frac{\mu}{\Lambda \sigma}\left[\ln \mathrm{I}-\frac{\mu+\Lambda \alpha}{\mu} \ln \left(\frac{\Lambda}{\mu}-\mathrm{I}\right)\right] .
$$

From Itô's formula and system (1.2), we have

$$
d V=f(V) d t+d B(t),
$$


where

$$
f(V)=\frac{1}{\sigma}\left[\beta-\frac{\mu\left(1+\alpha \eta^{-1}(V)\right)(\mu+\lambda)}{\Lambda-\mu \eta^{-1}(V)}-\frac{\sigma^{2}\left(\mu\left(\frac{\Lambda}{\mu}-\eta^{-1}(V)\right)^{2}-(\mu+\Lambda \alpha)\left(\eta^{-1}(V)\right)^{2}\right)}{2\left(1+\alpha \eta^{-1}(V)\right)^{2} \wedge}\right]
$$

with $I=\eta^{-1}(\mathrm{~V})$ since $\mathrm{R}^{*}=1$.

Now let $r=\eta(\chi)$, it is easy to get that

$$
\begin{aligned}
2 \int_{0}^{u} f(r) d r= & \frac{2}{\sigma^{2}} \int_{\eta^{-1}(0)}^{\eta^{-1}(u)}\left[\beta-\frac{\mu(1+\alpha \chi)(\mu+\lambda)}{\Lambda-\mu \chi}-\frac{\sigma^{2}\left(\mu\left(\frac{\Lambda}{\mu}-\chi\right)^{2}-(\mu+\Lambda \alpha) \chi^{2}\right)}{2(1+\alpha \chi)^{2} \Lambda}\right] \frac{\mu(1+\alpha \chi)}{(\Lambda-\mu \chi) \chi} d \chi \\
= & -\frac{2}{\sigma^{2}}\left[\left((\mu+\lambda) \frac{\mu^{2}}{\Lambda^{2}}+\frac{\sigma}{2}-\frac{\beta \mu}{\Lambda}\right) \ln \eta^{-1}(u)+\left(\frac{\beta(\mu+\Lambda \alpha)}{\Lambda}-(\mu+\lambda)\left(\frac{\mu^{2}}{\Lambda^{2}}-\alpha^{2}\right)+\frac{\sigma}{2}\right)\right. \\
& \left.\times \ln \left(\frac{\Lambda}{\mu}-\eta^{-1}(u)\right)+\frac{(\mu+\lambda)\left(\mu^{2}+\Lambda^{2} \alpha^{2}+2 \mu \Lambda \alpha\right)}{\Lambda\left(\Lambda-\mu \eta^{-1}(u)\right)}-\frac{\sigma \ln \left(1+\alpha \eta^{-1}(u)\right)}{2}\right]+M_{0},
\end{aligned}
$$

here $M_{0}$ is a constant. Then letting $u=\eta(\psi)$, we have

$$
\alpha(\mathrm{I})=\frac{\exp \left\{\mathrm{M}_{0}\right\}}{\sigma} \int_{\eta^{-1}(0)}^{\eta^{-1}(\mathrm{I})} \frac{(1+\alpha \psi)^{\frac{\sigma+1}{\sigma}}}{\psi^{\frac{2}{\sigma^{2}}\left((\mu+\lambda) \frac{\mu^{2}}{\Lambda^{2}}+\frac{\sigma}{2}-\frac{\beta \mu}{\Lambda}\right)+1}} \frac{\exp \left\{-\frac{2}{\sigma^{2}} \frac{(\mu+\lambda)\left(\mu^{2}+\Lambda^{2} \alpha^{2}+2 \mu \wedge \alpha\right)}{\Lambda(\Lambda-\mu \psi)}\right\}}{\left(\frac{\Lambda}{\mu}-\psi\right)^{\frac{2}{\sigma^{2}}\left(\frac{\beta(\mu+\Lambda \alpha)}{\Lambda}-(\mu+\lambda)\left(\frac{\mu^{2}}{\Lambda^{2}}-\alpha^{2}\right)+\frac{\sigma}{2}\right)+1}} \mathrm{~d} \psi .
$$

Notice that $\eta^{-1}(0) \in\left(0, \frac{\Lambda}{\mu}\right), \lim _{I \rightarrow+\infty} \eta^{-1}(I)=\frac{\Lambda}{\mu}, \lim _{I \rightarrow-\infty} \eta^{-1}(I)=0^{+}$. By Lemma 2.3 , one sees that

$$
\begin{aligned}
\alpha(+\infty) \leqslant & \frac{\exp \left\{\mathrm{M}_{0}\right\}}{\sigma}\left(1+\frac{\alpha \Lambda}{\mu}\right)^{\frac{\sigma+1}{\sigma}} \int_{\eta^{-1}(0)}^{\frac{\Lambda}{\mu}} \frac{1}{\psi^{\frac{2}{\sigma^{2}}\left((\mu+\lambda) \frac{\mu^{2}}{\Lambda^{2}}+\frac{\sigma}{2}-\frac{\beta \mu}{\Lambda}\right)+1}} \\
& \times \frac{\exp \left\{-\frac{2}{\sigma^{2}} \frac{(\mu+\lambda)\left(\mu^{2}+\Lambda^{2} \alpha^{2}+2 \mu \wedge \alpha\right)}{\Lambda(\Lambda-\mu \psi)}\right\}}{\left(\frac{\Lambda}{\mu}-\psi\right)^{\frac{2}{\sigma^{2}}\left(\frac{\beta(\mu+\Lambda \alpha)}{\Lambda}-(\mu+\lambda)\left(\frac{\mu^{2}}{\Lambda^{2}}-\alpha^{2}\right)+\frac{\sigma}{2}\right)+1}} \mathrm{~d} \psi \\
< & +\infty,
\end{aligned}
$$

and

$$
\begin{aligned}
\alpha(-\infty) & \leqslant-\frac{\exp \left\{M_{0}\right\}}{\sigma} \frac{1}{\eta^{-1}(0)^{\frac{2}{\sigma^{2}}\left((\mu+\lambda) \frac{\mu^{2}}{\Lambda^{2}}+\frac{\sigma}{2}-\frac{\beta \mu}{\Lambda}\right)}} \frac{\exp \left\{-\frac{2}{\sigma^{2}} \frac{(\mu+\lambda)\left(\mu^{2}+\Lambda^{2} \alpha^{2}+2 \mu \wedge \alpha\right)}{\Lambda\left(\Lambda-\mu \eta^{-1}(0)\right)}\right\}}{\left(\frac{\Lambda}{\mu}\right)^{\frac{2}{\sigma^{2}}\left(\frac{\beta(\mu+\Lambda \alpha)}{\Lambda}-(\mu+\lambda)\left(\frac{\mu^{2}}{\Lambda^{2}}-\alpha^{2}\right)+\frac{\sigma}{2}\right)+1}} \int_{0^{+}}^{\eta^{-1}(0)} \frac{1}{\psi} d \psi \\
& =-\infty .
\end{aligned}
$$

Similarly, we also obtain $\beta(+\infty)=+\infty, \beta(-\infty)=-\infty$. The proof of Theorem 2.5 is complete.

\section{Stochastic permanence}

Many results for permanence in mean has been obtained, however, we further investigate stochastic permanence in this section. Before analyzing the main results, we first give a useful definition as follows. 
Definition 3.1 ([15]). System (1.2) is said to be stochastically permanent, if for any $\varepsilon \in(0,1)$, there exist positive constants $\delta_{1}=\delta_{1}(\varepsilon)$ and $\delta_{2}=\delta_{2}(\varepsilon)$ such that

$$
\liminf _{t \rightarrow+\infty} \mathbb{P}\left\{I(t) \leqslant \delta_{1}\right\} \geqslant 1-\varepsilon, \quad \liminf _{t \rightarrow+\infty} \mathbb{P}\left\{I(t) \geqslant \delta_{2}\right\} \geqslant 1-\varepsilon,
$$

where $I(t)$ is an arbitrary solution of system (1.2) for any initial value $I(0) \in \mathbb{R}_{+}$.

Theorem 3.2. Let $\mathrm{I}(\mathrm{t})$ be the solution of system (1.2) with initial value $\mathrm{I}(0) \in\left(0, \frac{\Lambda}{\mu}\right)$. If $\mathrm{R}^{*}>1$ holds, then system (1.2) is stochastically permanent.

Proof. According to the Itô's formula and system (1.2), we get

$$
\begin{aligned}
d\left(I^{-\kappa}\right) & =-\kappa I^{-\kappa}\left(\frac{\beta\left(\frac{\Lambda}{\mu}-I\right)}{1+\alpha I}-(\mu+\lambda)-\frac{\sigma^{2}(\kappa+1)\left(\frac{\Lambda}{\mu}-I\right)^{2}}{2(1+\alpha I)^{2}}\right) d t-\frac{\sigma \kappa I^{-\kappa}\left(\frac{\Lambda}{\mu}-I\right)}{(1+\alpha I)} d B(t) \\
& =-\kappa I^{-\kappa}\left(\frac{\beta \Lambda}{\mu}-\mu-\lambda-\frac{\sigma^{2}(\kappa+1) \Lambda^{2}}{2 \mu^{2}}\right) d t+U(t) d t-\frac{\sigma \kappa I^{-\kappa}(\Lambda-\mu I)}{\mu(1+\alpha I)} d B(t),
\end{aligned}
$$

here $\kappa(0<\kappa<1)$ is a constant and

$$
\begin{aligned}
\mathrm{U}(\mathrm{t}) & =\kappa \mathrm{I}^{-\kappa}\left(\frac{\beta \Lambda}{\mu}-\frac{\beta(\Lambda-\mu \mathrm{I})}{\mu(1+\alpha \mathrm{I})}-\frac{\sigma^{2}(\kappa+1) \Lambda^{2}}{2 \mu^{2}}+\frac{\sigma^{2}(\kappa+1)(\Lambda-\mu \mathrm{I})^{2}}{2 \mu^{2}(1+\alpha \mathrm{I})^{2}}\right) \\
& \leqslant \frac{\beta \kappa(\Lambda \alpha+\mu) \Lambda^{1-\kappa}}{\mu^{2-\kappa}} .
\end{aligned}
$$

Set

$$
\phi=\kappa\left(\frac{\beta \Lambda}{\mu}-\mu-\lambda-\frac{\sigma^{2}(\kappa+1) \Lambda^{2}}{2 \mu^{2}}\right) .
$$

Take $\kappa$ small enough such that $\phi>0$. Furthermore, multiplying by $e^{\phi t}$ on the both sides of (3.1) and integrating from 0 to $t$ lead to

$$
\mathrm{I}^{-\kappa}(\mathrm{t})=e^{-\phi \mathrm{t}} \mathrm{I}^{-\kappa}(0)+\int_{0}^{\mathrm{t}} \mathrm{U}(\mathrm{r}) \mathrm{e}^{-\phi(\mathrm{t}-\mathrm{r})} \mathrm{dr}-\int_{0}^{\mathrm{t}} \frac{\sigma \kappa \mathrm{I}^{-\kappa}(\mathrm{r})(\Lambda-\mu \mathrm{I}(\mathrm{r}))}{\mu(1+\alpha \mathrm{I}(\mathrm{r}))} \mathrm{dB}(\mathrm{r}),
$$

then taking the expectation yields

$$
\begin{aligned}
\mathbb{E}\left[I^{-\kappa}(t)\right] & =e^{-\phi t} I^{-\kappa}(0)+\mathbb{E} \int_{0}^{t} U(r) e^{-\phi(t-r)} d r \\
& \leqslant I^{-\kappa}(0)+\frac{\beta \kappa(\Lambda \alpha+\mu) \Lambda^{1-\kappa}}{\phi \mu^{2-\kappa}}
\end{aligned}
$$

By virtue of $I(t) \in\left(0, \frac{\Lambda}{\mu}\right]$, using the Chebyshev inequality, we can get

$$
\begin{aligned}
\mathbb{P}\left\{\frac{\Lambda}{\mu} \geqslant I(t) \geqslant \delta_{2}\right\} & =\mathbb{P}\left\{\mathrm{I}(\mathrm{t}) \geqslant \delta_{2}\right\} \\
& =1-\mathbb{P}\left\{\delta_{2}^{-\kappa} \geqslant \mathrm{I}^{-\kappa}(\mathrm{t})\right\} \\
& \geqslant 1-\delta_{2}^{\kappa} \mathbb{E}\left[\mathrm{I}^{-\kappa}(\mathrm{t})\right] \\
& \geqslant 1-\delta_{2}^{\kappa}\left[\mathrm{I}^{-\kappa}(0)+\frac{\beta \kappa(\Lambda \alpha+\mu) \Lambda^{1-\kappa}}{\phi \mu^{2-\kappa}}\right] .
\end{aligned}
$$

Take $\delta_{2}$ such that $\delta_{2}^{\kappa}\left[I^{-\kappa}(0)+\frac{\beta \kappa(\Lambda \alpha+\mu) \Lambda^{1-k}}{\phi \mu^{2-\kappa}}\right]<\varepsilon$, we have $\liminf _{t \rightarrow+\infty} \mathbb{P}\left\{\mathrm{I}(\mathrm{t}) \geqslant \delta_{2}\right\} \geqslant 1-\varepsilon$. Similarly, we also 
have $\liminf _{t \rightarrow+\infty} \mathbb{P}\left\{\mathrm{I}(\mathrm{t}) \leqslant \delta_{1}\right\} \geqslant 1-\varepsilon$. The proof of Theorem 3.2 is complete.

\section{Conclusions and simulations}

This paper applies Feller's test and the canonical probability method which is different to Lyapunov method to investigate long-time dynamics of the stochastic system. We establish the threshold $\mathrm{R}^{*}=\frac{\beta \Lambda}{\mu(\mu+\lambda)}-\frac{\sigma^{2} \Lambda^{2}}{2 \mu^{2}(\mu+\lambda)}$ which completely determines disease extinction and stochastic permanence under stochastic perturbation. The main results are given as follows:

(I) When $R^{*}<1$ or $R^{*}=1$, the disease dies out with probability one.

(II) When $R^{*}>1$, the disease is stochastically permanent with probability one, which is different from the persistence in mean. Compare with previous study results $[4,6,21]$, the additional condition of $\sigma^{2} \leqslant \frac{\beta \mu}{\Lambda}$ is unnecessary. Therefore, we improve the main results of previous studies.

(a)

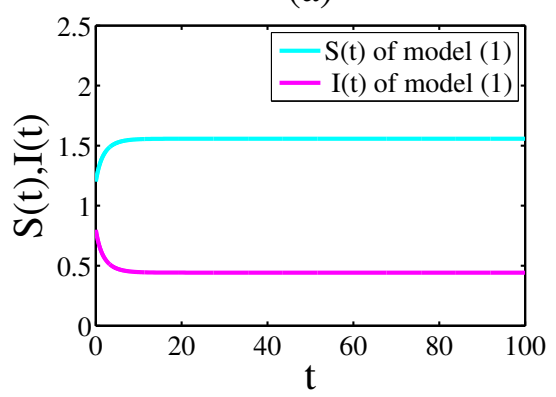

(b)

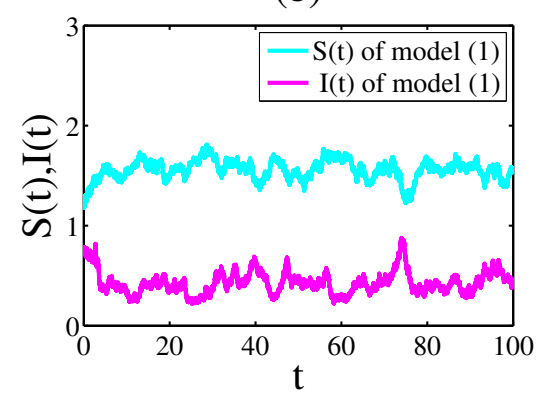

(c)

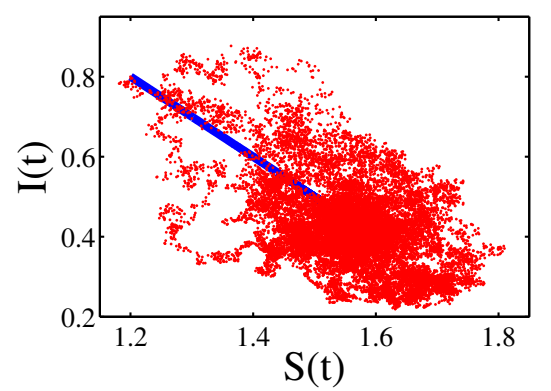

Figure 1: The simulation of the paths $S(t), I(t)$ for the SDE system (1.1), here (a) and (b) are time sequence diagrams, (c) stands for the phase portrait of (a) and (b). The blue trajectory and red dots in (c) are the deterministic system and the stochastic system of system (1.1), respectively. (a) $\sigma=0$, (b) $\sigma=0.15$.

Next, we present some numerical simulations to illustrate the theoretical results. Now we consider the following discrimination equations of system (1.1):

$$
\left\{\begin{array}{l}
S_{k+1}=S_{k}+\left(\Lambda-\mu S_{k}-\frac{\beta S_{k} I_{k}}{1+\alpha I_{k}}+\lambda I_{k}\right) \Delta t-\sigma \frac{S_{k} I_{k}}{1+\alpha I_{k}} \sqrt{\Delta t} \rho_{k}-\frac{\sigma^{2}}{2} \frac{S_{k} I_{k}}{1+\alpha I_{k}}\left(\rho_{k}^{2}-1\right) \Delta t \\
I_{k+1}=I_{k}+\left[\frac{\beta S_{k} I_{k}}{1+\alpha I_{k}}-(\mu+\lambda) I_{k}\right] \Delta t+\sigma \frac{S_{k} I_{k}}{1+\alpha I_{k}} \sqrt{\Delta t} \chi_{k}+\frac{\sigma^{2}}{2} \frac{S_{k} I_{k}}{1+\alpha I_{k}}\left(\chi_{k}^{2}-1\right) \Delta t
\end{array}\right.
$$

where $\rho_{k}, \chi_{k}(k=1,2, \cdots, n)$ stand for the Gaussian random variables $N(0,1)$ and time increment $\Delta t>0$. Take $S(0)=1.2, \mathrm{I}(0)=0.8, \Lambda=1, \beta=0.65, \lambda=0.3, \alpha=0.6, \mu=0.5, \Delta t=0.001$ and choose different values of $\sigma$ to explore the effect of the stochastic perturbation on dynamics for the SDE system (1.1).

In Figure $1(\mathrm{a})$, take $\sigma=0$, then $R^{*}=R=1.625>1$. As expected, Figure 1 (a) indicates the disease persists in real life. In Figure $1(b)$, take $\sigma=0.15$, then $R^{*}=1.5688>1$, it satisfies the condition in Theorem 3.2 and the previous work (A3), we can get that the disease will be stochastically permanent and permanent in mean with probability one. As expected, Figure 1 (b) supports our results of the Theorem 3.2 and the previous work (A3).

In Figure $2(a)$, take $\sigma=0$ and $R^{*}=R=1.625>1$, the result is similar to Figure 1 (a). In Figure $2(b)$, take $\sigma=0.7$, then $R^{*}=0.4<1$ holds, but we can see that $\sigma^{2}=0.49>\frac{\beta \mu}{\Lambda}=0.325$, it does not meet the conditions about the previous work (A2), so we can not make sure the disease will die out or persist by (A2). Synchronously, from Theorem $2.4, \mathrm{R}^{*}=0.4<1$ shows the disease will die out with probability one. As expected, Figure 2 (b) shows the disease dies out, which supports our results of Theorem 2.4. 
(a)

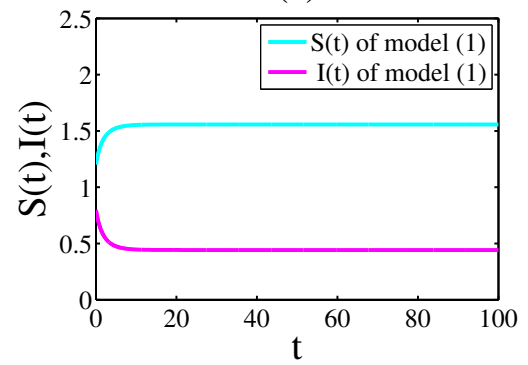

(b)

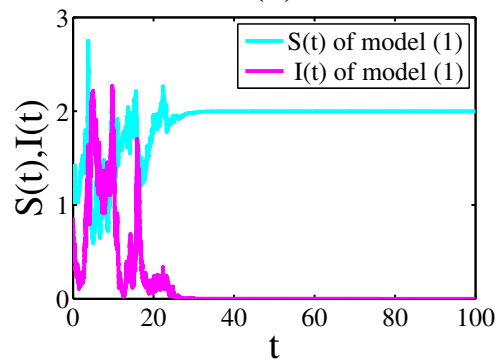

(c)

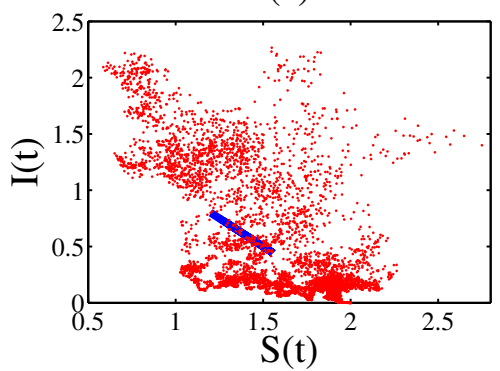

Figure 2: The simulation of the paths $S(t), I(t)$ for the SDE system (1.1), here (a) and (b) are time sequence diagrams, (c) denotes phase portrait of (a) and (b). The blue trajectory and red dots in (c) are the deterministic system and the stochastic system of system (1.1), respectively. (a) $\sigma=0$, (b) $\sigma=0.7$.

\section{Acknowledgment}

This work was supported by National Natural Science Foundation of China (11371230, 11561004), the SDUST Research Fund (2014TDJH102), Shandong Provincial Natural Science Foundation, China (ZR2015AQ001).

\section{References}

[1] Y.-L. Cai, Y. Kang, M. Banerjee, W.-M. Wang, A stochastic SIRS epidemic model with infectious force under intervention strategies, J. Differential Equations, 259 (2015), 7463-7502. 1

[2] T. Feng, X.-Z. Meng, L.-D. Liu, S.-J. Gao, Application of inequalities technique to dynamics analysis of a stochastic eco-epidemiology model, J. Inequal. Appl., 2016 (2016), 29 pages. 1

[3] S.-J. Gao, L.-S. Chen, J. J. Nieto, A. Torres, Analysis of a delayed epidemic model with pulse vaccination and saturation incidence, Vaccine, 24 (2006), 6037-6045. 1

[4] A. Gray, D. Greenhalgh, L. Hu, X. Mao, J. Pan, A stochastic differential equation SIS epidemic model, SIAM J. Appl. Math., 71 (2011), 876-902. 1, 1, 4

[5] Z.-X. Hu, W.-B. Ma, S.-G. Ruan, Analysis of SIR epidemic models with nonlinear incidence rate and treatment, Math. Biosci., 238 (2012), 12-20. 1

[6] C.-Y. Ji, D.-Q. Jiang, Threshold behaviour of a stochastic SIR model, Appl. Math. Model., 38 (2014), 5067-5079. 1, 1, 4

[7] Z. Jin, S.-P. Li, X.-G. Zhang, J.-P. Zhang, X.-L. Peng, Epidemiological modeling on complex networks, Complex Systems and Networks, Springer, Berlin, (2016). 1

[8] I. Karatzas, S. E. Shreve, Brownian motion and stochastic calculus, Springer, New York, (1988). 2, 2

[9] X.-P. Li, X.-Y. Lin, Y.-Q. Lin, Lyapunov-type conditions and stochastic differential equations driven by G-Brownian motion, J. Math. Anal. Appl., 439 (2016), 235-255. 1

[10] M. Liu, C.-Z. Bai, Analysis of a stochastic tri-trophic food-chain model with harvesting, J. Math. Biol., 73 (2016), $597-625$. 1

[11] Q. Liu, D.-Q. Jiang, N.-Z. Shi, T. Hayat, B. Ahmad, Stationary distribution and extinction of a stochastic SEIR epidemic model with standard incidence, Phys. A, 476 (2017), 58-69. 1

[12] L.-D. Liu, X.-Z. Meng, Optimal harvesting control and dynamics of two species stochastic model with delays, Adv. Difference Equ., 2017 (2017), 17 pages. 1

[13] X.-Z. Liu, P. Stechlinski, Infectious disease models with time-varying parameters and general nonlinear incidence rate, Appl. Math. Model., 36 (2012), 1974-1994. 1

[14] M. Liu, K. Wang, Stationary distribution, ergodicity and extinction of a stochastic generalized logistic system, Appl. Math. Lett., 25 (2012), 1980-1985. 1

[15] J.-L. Lv, K. Wang, Almost sure permanence of stochastic single species models, J. Math. Anal. Appl., 422 (2015), 675-683. 3.1

[16] H.-J. Ma, Y.-M. Jia, Stability analysis for stochastic differential equations with infinite Markovian switchings, J. Math. Anal. Appl., 435 (2016), 593-605. 1

[17] W.-B. Ma, M. Song, Y. Takeuchi, Global stability of an SIR epidemicmodel with time delay, Appl. Math. Lett., 17 (2004), 1141-1145. 1

[18] X.-R. Mao, Stochastic Differential Equations and Applications, Woodhead Publishing, Cambridge, (2007). 2.1

[19] X.-Z. Meng, Stability of a novel stochastic epidemic model with double epidemic hypothesis, Appl. Math. Comput., 217 (2010), 506-515. 1 
[20] X.-Z. Meng, L.-S. Chen, B. Wu, A delay SIR epidemic model with pulse vaccination and incubation times, Nonlinear Anal., Real World Appl., 11 (2010), 88-98. 1

[21] X.-Z. Meng, S.-N. Zhao, T. Feng, T.-H. Zhang, Dynamics of a novel nonlinear stochastic SIS epidemic model with double epidemic hypothesis, J. Math. Anal. Appl., 433 (2016), 227-242. 1, 1, 1, 4

[22] A.-Q. Miao, X.-Y. Wang, T.-Q. Zhang, W. Wang, B. G. S. A. Pradeep, Dynamical analysis of a stochastic SIS epidemic model with nonlinear incidence rate and double epidemic hypothesis, Adv. Difference Equ., 2017 (2017), 27 pages. 1

[23] A.-Q. Miao, J. Zhang, T.-Q. Zhang, B. G. S. A. Pradeep, Threshold dynamics of a stochastic SIR model with vertical transmission and vaccination, Comput. Math. Methods Med., 2017 (2017), 10 pages. 1

[24] J. J. Nieto, A. Ouahab, R. Rodríguez-López, Random fixed point theorems in partially ordered metric spaces, Fixed Point Theory Appl., 2016 (2016), 19 pages. 1

[25] X.-P. Sun, Y.-J. Wang, Stability analysis of a stochastic logistic model with nonlinear diffusion term, Appl. Math. Model., 32 (2008), 2067-2075. 2, 2

[26] X.-Y. Wang, W. Xu, X.-Z. Liu, Stochastic stability of stochastic switched epidemic models with constant and impulsive control schemes, Chaos Solitons Fractals, 78 (2015), 185-193. 1

[27] C.-Q. Xu, S.-L. Yuan, An analogue of break-even concentration in a simple stochastic chemostat model, Appl. Math. Lett., 48 (2015), 62-68.

[28] X.-H. Zhang, D.-Q. Jiang, A. Alsaedi, T. Hayat, Stationary distribution of stochastic SIS epidemic model with vaccination under regime switching, Appl. Math. Lett., 59 (2016), 87-93. 1

[29] T.-Q. Zhang, X.-Z. Meng, T.-H. Zhang, Global analysis for a delayed SIV model with direct and environmental transmissions, J. Appl. Anal. Comput., 6 (2016), 479-491. 1

[30] T.-Q. Zhang, X.-Z. Meng, T.-H. Zhang, Y. Song, Global dynamics for a new high-dimensional SIR model with distributed delay, Appl. Math. Comput., 218 (2012), 11806-11819.

[31] Z. Zhao, L.-S. Chen, X.-Y. Song, Impulsive vaccination of seir epidemic model with time delay and nonlinear incidence rate, Math. Comput. Simulation, 79 (2008), 500-510. 1

[32] Y. Zhao, W.-H. Zhang, Observer-based controller design for singular stochastic Markov jump systems with state dependent noise, J. Syst. Sci. Complex., 29 (2016), 946-958. 1 